\title{
Automatic Verification of Extensions of Hardware Descriptions
}

\author{
Hans Eveking \\ Institut für Datentechnik \\ Technische Hochschule Darmstadt \\ D-6100 Darmstadt, Fed. Rep. of Germany
}

\begin{abstract}
The extension of a hardware description is a description where all properties of the original one are maintained. The concept applies to a variety of design and verification problems including logic-verification and the verification of behavioral vs. structural descriptions. For a systematic discussion, several classes of temporal behavior and HDLconstructs for their representation are introduced. The verification tool LOVERT is surveyed which allows for the automatic verification of several types of extensions.
\end{abstract}

\section{Extensions of Descriptions}

In the following, the correctness of finite state systems is discussed in terms of an HDL-based hardware specification technique [3]. One hardware description, the specification, defines the meaning of correctness for another one, the implementation (Fig. 1).

$$
\begin{aligned}
& T(d 1) \longrightarrow \text { Description d1 } \leftarrow \text { Specification } \\
& T(d 2) \rightarrow \text { Description d2 } \leftarrow \text { Implementation }
\end{aligned}
$$

Fig. 1: Basic situation of a hardware specification technique

Hardware specification techniques are based on the concept of the axiomatiziation of HDL descriptions [4]. The axioms associated with a hardware description $d$ have two sources (i) the model-specific axioms which are due to the hardware-model involved, e.g., the axioms of boolean algebra, (ii) the description-specific axioms reflecting the properties of the specific description $\mathrm{d}$. The model- and description-specific axioms associated with a hardware description characterize a theory, i.e., a formal system of the predicate calculus.

Definition 1: A formula $A$ is a correct statement about a hardware description $\mathrm{d}$ iff it is a theorem of the associated theory $T(d)$, i.e.,

$$
\vdash_{T(d)} A
$$

The relationship between the specification and the implementation can be discussed in terms of the relationship between the associated theories. We study classes of a particularly simple relationship between two descriptions $\mathrm{d} 1$ and $\mathrm{d} 2$ :

Definition 2: A description $d 2$ is an extension of $d 1$ iff for all axioms of $T(d 1)$, i.e., for $A(T(d 1)$ ) holds

$$
\vdash_{T(d 2)} A(T(d 1))
$$


Note that on the basis of 1-2 all correct statements about $\mathrm{d} 1$ are correct about $\mathrm{d} 2$, too. The limitations of the concept of extension are due to the fact that the underlying modelling concepts of $\mathrm{d} 1$ and $\mathrm{d} 2$ have to be the same since the model-specific axioms of $T(d 1)$ have also to be theorems of $T(d 2)$ as required by 1-2. Problems involving temporal abstraction or value homomorphisms for which an interpretation of the theory $T(d 1)$ is necessary $[3,5]$ are not covered by the concept of extension.

In Section 2, the semantics of finite state systems is defined in terms of some concepts of mathematical systems theory [9]. HDL-representations of several types of temporal behavior are proposed. The HDL-constructs are taken from the CONLAN family of HDL's [8]. An axiomatiziaton of all HDL-constructs will be given.

In Sections 3-5, several types of extensions will be introduced, and proof-procedures will be discussed.

\section{Classes of Temporal Behavior}

We study systems that can be characterized by means of time-functions. A time-function represents the values that can be observed at a carrier, i.e., a point of observation. We consider time-functions to be functions from the set of natural numbers representing the time into some range, e.g., the set of boolean values $\{L, H\}$. Let $P$ be a set of $n$ time-functions $f_{1}, \ldots, f_{n} . P(t)$ denotes the

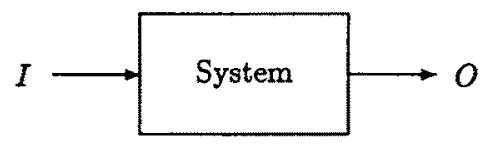

Fig. 2: A system with inputs and outputs

$n$-tupel $\left(f_{1}(t), \ldots, f_{n}(t)\right)$, i.e., the tupel of all $n$ values at point $t$ of time. $\left.P\left(<t_{1}, t_{2}\right\rangle\right)$ denotes the $\left(t_{2}-t_{1}+1\right)$-tupel of all $P(t)$ in the interval $t_{1} \leq t \leq t_{2}$.

A set $P$ of time-functions associated with a system can be partitioned into a set $I$ of input-functions and a set $O$ of output-functions (Fig. 2). The classification of the temporal behavior of a system is based on the question:

Which information about $I(<0, t>)$ and $O(<0, t-1>)$ determines $O(t)$ uniquely?

In the rest of this Section, HDL-constructs for the representation of three classes of behavior will be presented.

\subsection{Static behavior}

A system has static behavior iff $O(t)$ is determined uniquely by $I(t)$ for all $t$. A typical example is the behavior of an AND-gate (Fig. 3).

$L$ (low) and $H$ (high) are the boolean constants. Static behavior is described by means of connections to carriers of type btml (boolean terminal)

$$
\mathbf{g} \cdot \mathbf{\theta}
$$




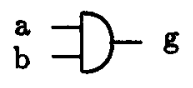

\begin{tabular}{||c|cccccc|}
\hline$t$ & 0 & 1 & 2 & 3 & 4 & $\ldots$ \\
\hline \hline$a(t)$ & L & L & H & L & L & $\ldots$ \\
\hline$b(t)$ & H & L & H & H & L & $\ldots$ \\
\hline$g(t)$ & L & L & H & L & L & $\ldots$ \\
\hline
\end{tabular}

Fig. 3: Static behavior of an AND-gate

with the meaning $\forall t: g(t)=e(t)$.

For the boolean functions \& (and), I (or), $I 1$ (exor) and * (not), corresponding boolean functions are defined in the predicate calculus, e.g.

$$
(\operatorname{and}(a, b)=r) \Longleftrightarrow(a=H) \wedge(r=b) \vee(a=L) \wedge(r=L) \text {. }
$$

The meaning of an HDL-expression is defined by means of a time-function, too. For instance, the time-function $\lambda(t)($ and $(a(t), b(t)))$ is associated with the boolean expression a $\&$ b of Fig. 3 . As a result, the meaning of the statement $\mathrm{g}=\mathrm{a} \& \mathrm{~b}$ of Fig. 3 is

$$
\forall t: g(t)=(\lambda(t)(\operatorname{and}(a(t), b(t))))(t)=\operatorname{and}(a(t), b(t)) .
$$

\subsection{Transitional behavior}

A system has transitional behavior iff $O(t)$ is uniquely determined by $I(t-1)$ and $O(t-1)$ for $0<t$. The behavior of a Moore-machine [6] is an example of transitional behavior. This type of behavior is described by conditional transfers (Fig. 4) into carriers of type budvl (boolean unit delay variable). If the transfer condition $a$ is $\mathrm{H}$ at point $t-1$ then the value of $x$ at point $t$ becomes

IF a THEN $x<-y$ ENDIF

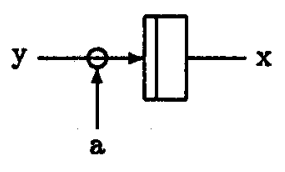

\begin{tabular}{||c|ccccccc|}
\hline$t$ & 0 & 1 & 2 & 3 & 4 & 5 & $\ldots$ \\
\hline \hline$a(t)$ & L & L & H & L & L & H & $\ldots$ \\
\hline$y(t)$ & H & H & H & L & L & L & $\ldots$ \\
\hline$x(t)$ & L & L & L & H & H & H & $\ldots$ \\
\hline
\end{tabular}

Fig. 4: Transitional behavior of a transfer

the value of $y(t-1)$; otherwise, the old value of $x$ is maintained. A default value $L$ is assumed for point 0 of time.

The semantics of a conditional transfer is thus

$$
\begin{aligned}
& x(0)=L, \\
& \forall t:(0<t) \wedge(a(t-1)=H) \Rightarrow(x(t)=y(t-1)), \\
& \forall t:(0<t) \wedge(a(t-1)=L) \Rightarrow(x(t)=x(t-1)) .
\end{aligned}
$$

There may be several conditional transfers into one carrier. Assume n conditional transfers into the carrier $\mathbf{x}$ :

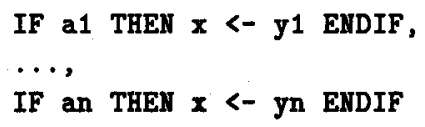


If transfer collisions are excluded then the meaning is:

$$
\begin{aligned}
& x(0)=L, \\
& \forall t:(0<t) \wedge(a 1(t-1)=H) \Rightarrow(x(t)=y 1(t-1)), \\
& \ldots \\
& \forall t:(0<t) \wedge(a n(t-1)=H) \Rightarrow(x(t)=y n(t-1)), \\
& \forall t:(0<t) \wedge(a 1(t-1)=L) \wedge \ldots \wedge(\operatorname{an}(t-1)=L) \Rightarrow(x(t)=x(t-1)) .
\end{aligned}
$$

If a transfer condition or source expression is a boolean expression, e.g.,

IF $a$ \& $b$ THEN $x<-y$ ENDIF

then an anonymous time-function is associated with the boolean expression (Section 2.1). The time-functions of all carriers are thus bound to point $t-1$ of time. In the example, we obtain

$$
\begin{aligned}
& x(0)=L, \\
& \forall t:(0<t) \wedge(\operatorname{and}(a(t-1), b(t-1))=H) \Rightarrow(x(t)=y(t-1)), \\
& \forall t:(0<t) \wedge(\operatorname{and}(a(t-1), b(t-1))=L) \Rightarrow(x(t)=x(t-1)) .
\end{aligned}
$$

\subsection{Quasi-transitional behavior}

A system has quasi-transitional behavior iff $O(t)$ is uniquely determined by $I(t)$ und $O(t-1)$ for $0<t$. An example is the behavior of a latch (Fig. 5 ) described by a conditional assignment to a

IF a THEN $r:=y$ ENDIF

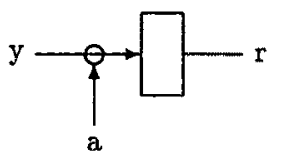

\begin{tabular}{||c|ccccccc|}
\hline$t$ & 0 & 1 & 2 & 3 & 4 & 5 & $\ldots$ \\
\hline \hline$a(t)$ & L & L & H & L & L & H & $\ldots$ \\
\hline$y(t)$ & H & H & H & L & L & L & $\ldots$ \\
\hline$r(t)$ & L & L & H & H & H & L & $\ldots$ \\
\hline
\end{tabular}

Fig. 5: Quasi-transitional behavior of a latch

carrier of type bvarl (boolean variable). Note that in the example of Fig. 5, the output $r$ follows the input $y$ directly at points 2 and 5 of time.

The meaning of the conditional assignment of Fig. 5 is defined by

$$
\begin{aligned}
& x(0)=L, \\
& \forall t:(0<t) \wedge(a(t)=H) \Rightarrow(x(t)=y(t)), \\
& \forall t:(0<t) \wedge(a(t)=L) \Rightarrow(x(t)=x(t-1)) .
\end{aligned}
$$

The input/output behavior of an automaton of Mealy-Type [6] is also quasi-transitional.

\section{Static Descriptions vs. Static Descriptions}

The first type of extensions applies to situations where the specification as well as the implementation are given by static descriptions.

Definition 3: A description is called static iff all carriers have static behavior. 

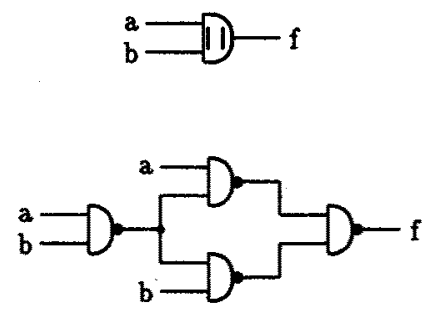

Fig. 6: An example of logic-verification

EXOR-function is implemented by means of a network of NAND-gates. To prove the correct implementation, the boolean terms of the output $f$ are derived for the specification as well for the implementation; then the equivalence $\simeq$ of both terms is shown:

$$
a \mid 1 b \simeq \sim(a+\sim(a \& b)) \& \sim(b \&-(a+b)))
$$

Efficient procedures for the equivalence-proof of complex boolean expressions are available due to the work of Bryant [2] and Madre/Billon [7]. Since most HDL's provide bit-vectors as a basic type for the convenient description of complex circuits, the problem of logic-verification involves also the equivalence-proof of vector-expressions.

Definition 4: Two vector-expressions are equivalent, $a \cong b$, iff for all elements $i$ holds $a[i] \simeq$ $b[i]$.

The semantics of vectors and vector-operations can be defined by means of lists and list-operations [4]. Three examples of increasing complexity are shown in Fig. 7. The first problem is easily solved if the commutativity of the boolean and-function is extended for vector-functions. In the

$$
\begin{array}{cccc}
a[1: 8] \& b[1: 8] & \cong & b[1: 8] \& a[1: 8] \\
\operatorname{adc}(a[1: 8], a[1: 8], 0) & \cong & a[1: 8] * 0 \\
\operatorname{gt}(a, b) & \cong & -(\operatorname{adc}(0 \# a, 1 *-b, 0)[2])
\end{array}
$$

Fig. 7: Three examples of equivalent vector-expressions

second problem, the function adc is used which adds two $n$-bit vectors and a carry-input returning a normalized $n+1$-bit vector $1: n+1$. The left-most bit is the most-significant bit. For instance,

$$
\operatorname{adc}(a[1: 8], b[1: 8], c)
$$

is a 9-bit vector; the carry-output is adc $(a[1: 8], b[1: 8], c)[1]$. The second equivalence of Fig. 7 is based on the fact that a plus a is equivalent to a multiplication of a by 2 , i.e., a left-shift of 
a or the concatenation \# of and $0 .{ }^{1}$ The third problem is even more difficult: in order to compare two vectors $a$ and $b, b$ is subtracted from $a$ adding the complement of $b$ to $a$; the most-significant bit of the sum has to be inverted.

To address such a variety of proof-complexity, the LOVERT approach [1] follows a two-step procedure:

- Step 1: two expressions are rewritten using a number of rewrite-rules. If the rewriting results in textually identical expressions then the equivalence is proven (Fig. 7). An example of a

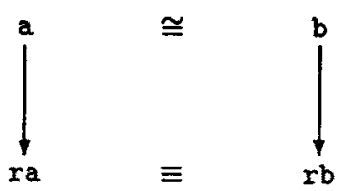

Fig. 8: Transformation of an equivalence-proof into an identity-proof

rewrite-rule is the concatenation of the adc-function: two concatenated adders

$$
\begin{aligned}
& \operatorname{adc}(x, y, \operatorname{adc}(v[1: n], v[1: n], \operatorname{cin})[1]) \# \\
& \operatorname{adc}(v[1: n], v[1: n], \operatorname{cin})[2: n]
\end{aligned}
$$

are equivalent to one adder with catenated inputs:

$$
\operatorname{adc}(x \# v[1: n], y \# w[1: n], \operatorname{cin}) \text {. }
$$

- Step 2: if the rewrite-technique fails, the vector-expressions are compiled into the basic boolean functions and, or and not. Vector expressions are sliced into single bits. The Madre/Billon tautology checking technique [7] is then applied (Fig. 9). Since the rewriterules used in Step 1 are not confluent, the second step ensures the completeness of the approach.

The following table shows the cpu-time of a SPARC-station needed to solve the third problem of Fig. 7 depending on wordlength:

\begin{tabular}{|r||c|c|c|c|c|}
\hline Wordlength & 8 & 16 & 32 & 64 & 128 \\
\hline CPU-time & $0.8 \mathrm{sec}$. & $0.9 \mathrm{sec}$. & $1.0 \mathrm{sec}$. & $1.8 \mathrm{sec}$. & $3.4 \mathrm{sec}$. \\
\hline
\end{tabular}

The two-step procedure has a significant advantage in a situation where a design error is detected. The behavior of a verification tool in an error situation is an important aspect for its acceptance by a designer. In the example of Fig. 10, a 16-bit adder is implemented by means of four 4-bit adders; however, the carry chain is broken at the carry input of the instance a2 since the carry input is erroneously set to 0 rather than to the carry output a1.co of the first adder. If the implementation of Fig. 10 is compared with the specification of an 16-bit adder

$$
\operatorname{adc}(a[1: 16], b[1: 16], 0)
$$

\footnotetext{
${ }^{1}$ For convenience, the HDL constants 0 and 1 are overloaded and represent the boolean constants. L and H as well as the integers 0 and 1
} 


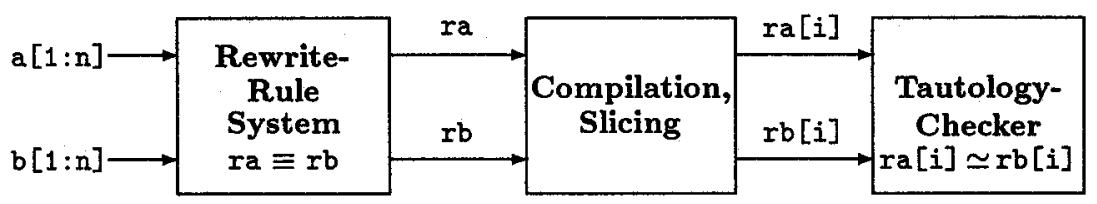

Fig. 9: Equivalence-proof of vector expressions

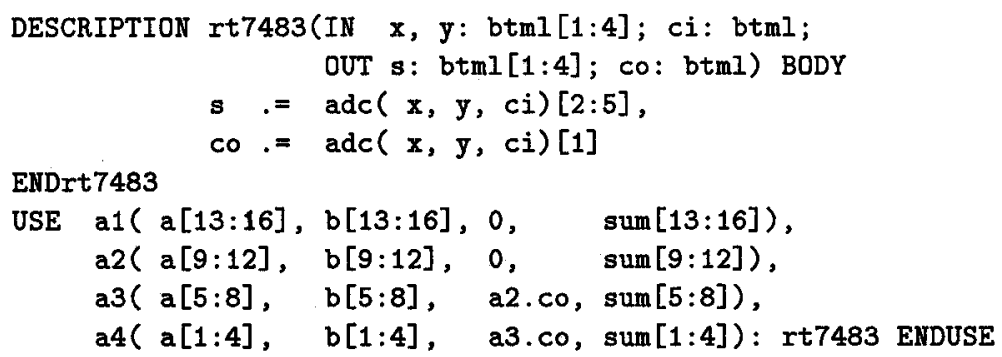

Fig. 10: Incorrect implementation of a 16-bit adder

then the response of LOVERT is the simplified expression of the implementation:

$$
\operatorname{adc}(a[1: 12], b[1: 12], 0) \# \operatorname{adc}(a[13: 16], b[13: 16], 0)[2: 5]
$$

The rewrite-rule system is able to simplify the expression for the three correctly chained 4-bit adders applying the simplification-rule for adders shown above; this results in an 12-bit adder which is concatenated with the last (and erroneously uncoupled) 4-bit adder. The expression gives thus a hint to the place where the problem is located.

This example shows also that LOVERT is able to cope with the problems involved in the aggregation of bit-sliced circuits.

\section{Transitional vs. Structure-Oriented Static/ Transitional Descriptions}

The second type of extensions applies to systems with sequential behavior.

Definition 5: A description is called transitional iff all carriers have transitional behavior.

Clearly, a description consisting of a collection of conditional transfers is transitional.

The main purpose of a transitional description is to display which transfers take place under which mutually exclusive conditions. The class of transitional descriptions comprises representations of simple state-diagrams as well as specifications of complex microprograms.

A further class of descriptions is the classical implementation of finite state machines as a composition of a transitional and a static subsystem (Fig. 11).

Definition 6: A static/transitional description is a combination of a static and of a transitional description. 


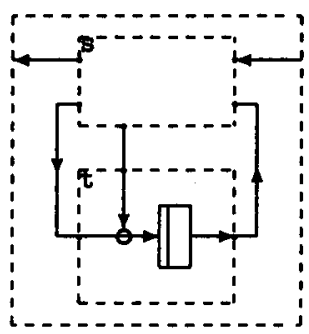

Fig. 11: Mixed transitional/static description

Definition 7: A structure-oriented description is a static/transitional description where each carrier of the transitional part occurs exactly once as destination.

Structure-oriented descriptions are amenable to an implementation by hardware since each transfer and each connection refers to one substructure.

We will now discuss a situation where the specification is a transitional description and where the implementation is a structure-oriented static/transitional description.

Assume several transfers into one carrier $\mathrm{x}$ in the transitional description d1:

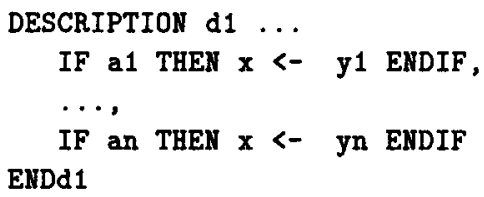

According to Definition 7, there is only one transfer into $x$ in the description d2:

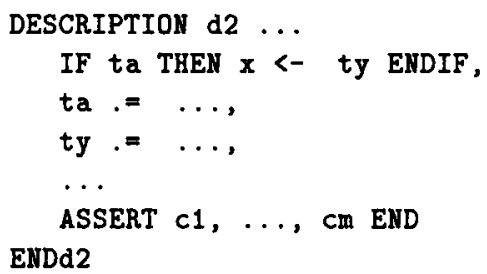

The assertions $c 1, \ldots \mathrm{cm}$ are used to specify don't care conditions, e.g., to exclude unreachable states or input combinations which must not occur.

To demonstrate 1-2, it must be proven for each transfer

$$
\text { IF ai THEN } x<-\quad y i \text { ENDIF }
$$

of $d 1$ that

1. the condition ai of $d 1$ implies the condition ta of d2:

$$
\vdash_{T(d 2)} \forall t:(c 1(t)=H) \wedge \ldots \wedge(c m(t)=H) \wedge(a i(t)=H) \Rightarrow(t a(t)=H)
$$


2. the condition ai implies the equality of the sources $y i$ and ty, respectively:

$$
\vdash_{T(d 2)} \forall t:(c 1(t)=H) \wedge \ldots \wedge(c m(t)=H) \wedge(a i(t)=H) \Rightarrow(t y(t)=y i(t)) .
$$

3 . in the storage situation (see 2-2) the content of $x$ remains unchanged if there is no ai active:

$$
\begin{gathered}
\vdash_{T(d 2)} \forall t:(c 1(t)=H) \wedge \ldots \wedge(c m(t)=H) \wedge(a 1(t)=L) \wedge \ldots \wedge(a n(t)=L) \Rightarrow \\
(\operatorname{ta}(t)=L) \vee(\operatorname{ty}(t)=x(t)) .
\end{gathered}
$$

Note that 4-1, 4-2 and 4-3 refer to the point $t$ of time only and amounts thus to an equivalence proof of vector-expressions. The LOVERT system is also able to cope with this type of extensions. Typical examples of application are the proof of the correct implementation of microprograms by means of a register-bus structure and a microprogram-sequencer including a ROM. An average verification time of $1-2 \mathrm{sec}$. per transfer on a SPARC-station was observed. The correct implementation of a complete microprogram is thus proven in a few minutes.

\section{Transitional vs. Quasi-Transitional Descriptions}

In the third type of extensions, transitional ("buffered") behavior of transfers is implemented by means of quasi-transitional ("unbuffered") assignments.

An example-specification of transitional-behavior is given in Fig. 12.

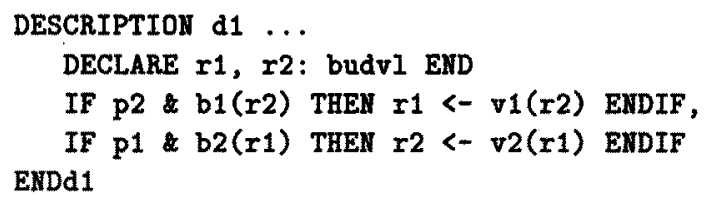

Fig. 12: Specification of transitional behavior

The implementation by means of a quasi-transitional description using conditional assignments is shown in Fig. 13.

Definition 8: A description is called quasi-transitional iff all carriers have quasi-transitional behavior.

As an example of the proof of 1-2, we study one of the axioms associated with $r 1$ of the specification (see 2-1):

$$
\forall t:(0<t) \wedge(p 2(t-1)=H) \wedge(b 1(r 2(t-1))=H) \Rightarrow(r 1(t)=v 1(r 2(t-1))) .
$$

The corresponding axiom of the implementation of $r 1$ is according to 2-3

$$
\forall t:(0<t) \wedge(p 1(t)=H) \wedge(b 1(r 2(t))=H) \Rightarrow(r 1(t)=v 1(r 2(t))) .
$$

In order to accommodate 5-1 and 5-2, we have to require:

$$
\forall t:(0<t) \wedge(p 1(t)=H) \Rightarrow(p 2(t-1)=H)
$$

and

$$
\forall t:(0<t) \wedge(p 1(t)=H) \Rightarrow(r 2(t)=r 2(t-1))
$$



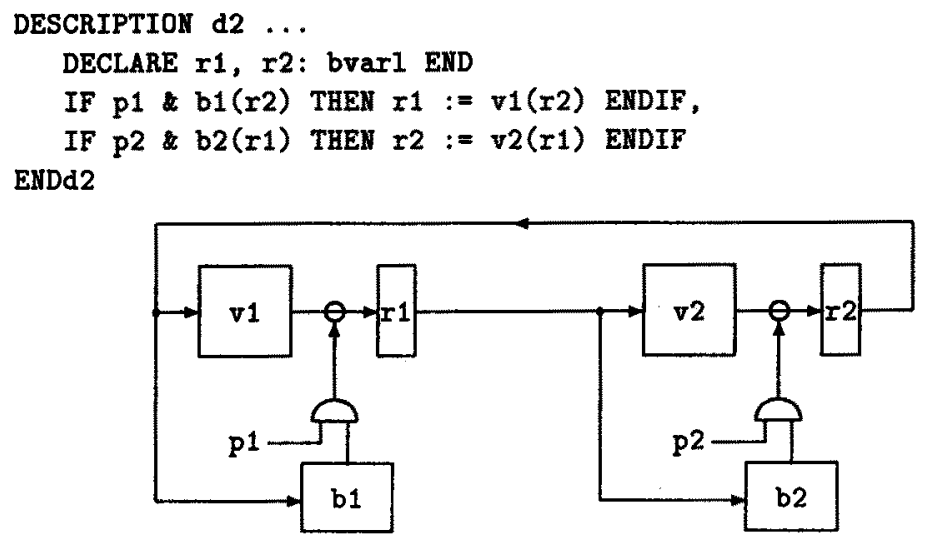

Fig. 13: Quasi-transitional implementation

The axioms associated with $r 2$ are (see 2-3)

$$
\begin{aligned}
& r 2(0)=L, \\
& \forall t:(0<t) \wedge(p 2(t)=H) \wedge(b 2(r 1(t))=H) \Rightarrow(r 2(t)=v 2(r 1(t))), \\
& \forall t:(0<t) \wedge((p 2(t)=L) \vee(b 2(r 1(t))=L)) \Rightarrow(r 2(t)=r 2(t-1)) .
\end{aligned}
$$

Hence $5-4$ is satisfied by

$$
\forall t:(0<t) \wedge(p 1(t)=H) \Rightarrow(p 2(t)=L) .
$$

Considering 5-3 and 5-5 we see that the correct implementation is ensured by a two-phase clock with the two phases $\mathrm{p} 1$ and $\mathrm{p} 2$. An example behavior is given in Fig. 14.

\begin{tabular}{||c|cccccc|}
\hline$t$ & 0 & 1 & 2 & 3 & 4 & $\ldots$ \\
\hline \hline$p 1(t)$ & $\mathrm{H}$ & $\mathrm{L}$ & $\mathrm{H}$ & $\mathrm{L}$ & $\mathrm{H}$ & $\ldots$ \\
\hline$p 2(t)$ & $\mathrm{L}$ & $\mathrm{H}$ & $\mathrm{L}$ & $\mathrm{H}$ & $\mathrm{L}$ & $\ldots$ \\
\hline
\end{tabular}

Fig. 14: Two-phase clock

As a result, it was shown that the quasi-transitional description of Fig. 13 has also transitional behavior. Note that the specification $\mathrm{d} 2$ of Fig. 12 is an abstraction from real hardware: there is no circuit that corresponds, e.g., to the expression $\mathrm{p} 2$ b1 (r2).

\section{Summary}

The relatively simple concept of extension covers a number of relevant design and verification problems including the problem of logic-verification, the problem of the correct implementation of state-diagrams by means of structural resources, and the problem of the implementation of state-diagrams by means of two-phase clocked systems. 
The introduction of several classes of temporal behavior represented by appropriate HDL-constructs allows for a systematic discussion of several types of extensions.

HDL-based hardware specification techniques offer a number of advantages: (i) hardware is represented in a convenient and user-friendly way, (ii) relevant classes of verification problems can be discussed in terms of the relationship between different forms of HDL-descriptions, (iii) the designer does not need proof-expertise since specialized verification tools support fully automatic verification.

The proof problems are complicated by the vector-functions provided by most HDL's for the compact representation of hardware. The LOVERT-approach proposes a combination of rewrite and tautology-checking techniques which makes the automatic verification of complex designs feasible.

\section{References}

[1] A. Bartsch, H. Eveking, H.-J. Faerber, M. Kelelatchew, J. Pinder, and U. Schellin. LOVERT - a logic verifier of register-transfer level descriptions. In L. Claesen, editor, Proc. IMEC-IFIP Workshop on Applied Formal Methods for Correct VLSI Design, pages 522-531, 1989.

[2] R.E. Bryant. Graph-based algorithms for boolean function manipulation. IEEE C-95, 677-691, 1986.

[3] H. Eveking. The application of CHDL's to the abstract specification of hardware. In Koomen/Moto-Oka, editor, Proc. CHDL' 85 (Tokio), pages 167-178, North-Holland, 1985.

[4] H. Eveking. Axiomatizing hardware description languages. International Journal of VLSI Design, 1990.

[5] H. Eveking. Formal verification of synchronous systems. In G. Milne and P.A. Subrahmanyam, editors, Formal Aspects of VLSI Design, pages 137-152, North-Holland, 1985.

[6] J. Hartmanis and R.E. Stearns. Algebraic Structure Theory of Sequential Machines. Prentice Hall, 1966.

[7] J.C. Madre and J.P. Billon. Proving circuit correctness by formally comparing their expected and extracted behavior. Proc. 25th Design Autom. Conf., 205-210, 1988.

[8] R. Piloty, M. Barbacci, D. Borrione, D. Dietmeyer, F. Hill, and P. Skelly. CONLAN Report. Springer-Verlag, Berlin Heidelberg New-York Tokio, 1983.

[9] T. G. Windeknecht. General Dynamical Processes. Academic Press, 1971. 